Revue d'histoire de l'Amérique française

REVUE D.HISTOIRE DE L'AMÉRIQUE FRANÇAISE

\title{
Quelques influences françaises sur l'historiographie religieuse du Québec des dernières décennies
}

\section{Jean Roy}

Volume 51, numéro 2, automne 1997

Les pratiques de l'histoire de l'Amérique française depuis 50 ans

URI : https://id.erudit.org/iderudit/305651ar

DOI : https://doi.org/10.7202/305651ar

Aller au sommaire du numéro

Éditeur(s)

Institut d'histoire de l'Amérique française

ISSN

0035-2357 (imprimé)

1492-1383 (numérique)

Découvrir la revue

Citer cet article

Roy, J. (1997). Quelques influences françaises sur l'historiographie religieuse du Québec des dernières décennies. Revue d'histoire de l'Amérique française, 51(2), 301-316. https://doi.org/10.7202/305651ar
Résumé de l'article

Grâce aux apports de la sociologie religieuse et de l'histoire des mentalités, l'historiographie religieuse française des cinquante dernières années s'est graduellement renouvelée. Est-ce que celle du Québec s'en est inspirée et quelles tendances a-t-elle suivies? La réponse, positive, à ces questions repose sur l'examen de quatre points : sociologie religieuse et recrutement, déchristianisation appréhendée et christianisation encadrée, romanisation du clergé et ultramontanisme religieux, religion populaire.
Tous droits réservés @ Institut d'histoire de l'Amérique française, 1997
Ce document est protégé par la loi sur le droit d'auteur. L'utilisation des services d’Érudit (y compris la reproduction) est assujettie à sa politique d'utilisation que vous pouvez consulter en ligne.

https://apropos.erudit.org/fr/usagers/politique-dutilisation/ 


\title{
QUELQUES INFLUENCES FRANÇAISES SUR L'HISTORIOGRAPHIE RELIGIEUSE DU QUÉBEC DES DERNIÈRES DÉCENNIES
}

\author{
JEAN ROY \\ Centre interuniversitaire d'études québécoises \\ Université du Québec à Trois-Rivières*
}

\section{RÉSUMÉ}

Grâce aux apports de la sociologie religieuse et de l'histoire des mentalités, l'historiographie religieuse française des cinquante dernières années s'est graduellement renouvelée. Est-ce que celle du Québec s'en est inspirée et quelles tendances at-elle suivies? La réponse, positive, à ces questions repose sur l'examen de quatre points: sociologie religieuse et recrutement, déchristianisation appréhendée et christianisation encadrée, romanisation du clergé et ultramontanisme religieux, religion populaire.

\section{ABSTRACT}

With the contribution of religious sociology and of the history of mentalities, the French religious historiography of the last fifty years renewed itself to a great extent. Did Quebec's religious historiography inspire itself from it and what trends did it follow? The answer, which is positive, to these questions relies on the examination of four elements: religious sociology and recruiting, apprehended dechristianization and supervised christianization, the romanizing of the clergy and religious ultramontanism, and popular religion.

Pour faire le point sur l'état actuel de l'historiographie religieuse québécoise, nous disposons de deux bilans récents. Dans le premier, Brigitte Caulier ${ }^{1}$ examine le sentiment religieux. Elle met en perspective les tendances nouvelles créées par les conjonctures religieuse et nationale qui sont à la naissance des objets de recherche sur le catholicisme québécois depuis 1960. L'épiscopat s'alarmait de la désertion des églises et chargea la commission Dumont d'en étudier le mouveconseils.

* Mes remerciements vont à René Hardy pour sa lecture du texte ainsi que pour ses

1. Brigitte Caulier, «Le sentiment religieux», dans Pierre Hurtubise et Jean-Marie LeBlanc, dir., Status Quaestionis (Ottawa, Université Saint-Paul, 1994), 47-59. 
ment. Celle-ci plaça le laïque au cœur de sa réflexion, donnant de la sorte une orientation aux études universitaires dans plusieurs disciplines. Alors que les sociologues établissaient des diagnostics, les ethnologues battaient la campagne pour recueillir des informations de la part d'une espèce en voie de disparition: le pratiquant. La religion populaire, un concept qui allait faire fortune, date de ces années. D'autres chercheurs s'intéressaient au patrimoine religieux. Enfin, après s'être beaucoup occupés des institutions et des idéologies, les historiens commencèrent à se tourner vers les formes obligatoires et surérogatoires de la pratique, ainsi que vers l'encadrement des laïques par les clercs. Les recherches des dix dernières années s'inscrivent dans cette continuité: une meilleure définition des conjonctures, l'enseignement religieux et le catéchisme en sont des sujets parmi d'autres.

Le second bilan est dû à Guy Laperrière ${ }^{2}$. Lui aussi s'interroge sur la place des laïques et des fidèles dans l'historiographie et il répartit en trois périodes la production livresque des cinquante dernières années. Dans la première, la religion et l'Église catholique sont le centre et le moteur de l'histoire ainsi qu'une caractéristique de la société canadienne-française. Lui succède, à partir de 1960, une période de contestation sociale et de sécularisation. L'écriture de l'histoire sort renouvelée de son transfert de domination, passant des clercs aux laïques. Enfin, une troisième période s'ouvre avec les débuts des années 1980: le fait religieux est soumis à des éclairages neufs venant de plusieurs disciplines.

Ces deux états sont largement complémentaires et ils enseignent adéquatement sur la production. Si bien que le dessein d'en ajouter un autre peut sembler superflu. Le risque évident, sinon le piège, serait de répéter. Mais alors, comment être utile et, si possible, intéressant? La réponse à cette question est venue en relisant un autre bilan historiographique rédigé par les historiens Claude Langlois et André Vauche $z^{3}$ sur l'histoire religieuse française au cours des cinquante dernières années. Il m'a semblé alors qu'une comparaison pouvait être

2. Guy Laperrière, «L'évolution de l'histoire religieuse au Québec depuis 1945: le retour du pendule?», dans Yves Roby et Nive Voisine, dir., Érudition, humanisme et savoir (Sainte-Foy, Les Presses de l'Université Laval, 1996), 331-348. Actes du colloque en l'honneur de Jean Hamelin.

3. Claude Langlois et André Vauchez, «L'histoire religieuse», dans François Bédarida, dir., L'histoire et le métier d'historien en France, 1945-1995 (Paris, Éditions de la Maison des sciences de l'homme, 1995), 313-323; tout aussi suggestif, mais dans une perspective différente quoique des recoupements existent, est le point fait par Michel Lagrée, "Histoire religieuse, histoire culturelle», dans Jean-Pierre Rioux et Jean-François Sirinelli, Pour une histoire culturelle (Paris, Seuil, 1997), 387-406. 
établie entre les historiographies française et québécoise afin de déterminer des zones d'influence. Plus précisément, je pense ici aux marques de l'historiographie française sur nos travaux et je vais tenter de démontrer que les travaux universitaires en sont beaucoup empreints ${ }^{4}$.

Ma démonstration passe d'abord par un bref résumé des tendances de l'historiographie française emprunté à l'article de Langlois et de Vauchez. Ceux-ci font ressortir les apports de la sociologie religieuse et de l'histoire des mentalités. Puis, je justifierai mon propos en m'appuyant sur quatre sujets: sociologie religieuse et recrutement, déchristianisation appréhendée et christianisation encadrée, romanisation du clergé et ultramontanisme religieux, religion populaire.

\section{A) LES CHANGEMENTS DANS L'ÉCRITURE DE L'HISTOIRE} DE L'HEXAGONE

Publiés à partir de 1962, les travaux de Toussaert, Pérouas et Marcilhacy ${ }^{5}$ montrent que l'appel de Gabriel Le Bras a été entendu. Grâce à la sociologie religieuse, l'histoire des institutions et des doctrines avait fait place à l'Histoire vécue du peuple chrétien ${ }^{6}$ pour prendre le titre d'un livre de synthèse. L'inspiration est venue également des travaux du chanoine Boulard', des interrogations et de l'intérêt que la lecture de ses cartes suscita pour «une géographie de la différence», pour la quantification de la pratique et pour la féconde problématique de la déchristianisation. Parmi les nouveaux chantiers ouverts par les enquêtes de sociologie religieuse, mentionnons ceux qui por-

4. Cette position va à l'encontre de celle de Guy Laperrière, loc. cit. Son bilan historiographique se fonde exclusivement sur la production livresque, négligeant volontairement les ouvrages collectifs ainsi que les articles de revues. Pour ma part, je ne fais pas de distinction entre les types de travaux. À telle enseigne qu'un livre de synthèse comme l'Histoire du catholicisme québécois dirigé par Nive Voisine doit être mis sur le même pied que l'ensemble de la production historique: ainsi, Lucien Lemieux et Nive Voisine ont largement mis à profit une documentation qui jusque-là n'avait pas été exploitée. Cela donne une teinte de nouveauté à l'œuvre et autorise à l'insérer dans le corpus.

5. J. Toussaert, Le sentiment religieux en Flandre à la fin du Moyen-Âge (Paris, Plon, 1963); Louis Pérouas, Le diocèse de La Rochelle de 1648 à 1724. Sociologie et pastorale (Paris, SEVPEN, 1964), 532 p.; Christiane Marcilhacy, Le diocèse d'Orléans sous l'épiscopat de M $^{r}$ Dupanloup, 1849-1878. Sociologie religieuse et mentalités collectives (Paris, Plon, 1962), 592 p. À quoi j'ajoute un ouvrage de moindre notoriété: Jeanne Ferté, La vie religieuse dans les campagnes parisiennes, 1622-1695 (Paris, Vrin, 1962), $453 \mathrm{p}$.

6. Jean Delumeau, dir., Histoire vécue du peuple chrétien (Toulouse, Privat, 1979), 2 tomes, $481 \mathrm{p}$.

7. Fernand Boulard, Premiers itinéraires en sociologie religieuse (Paris, Les Éditions ouvrières, 1954), $159 \mathrm{p}$. 
tèrent sur le recrutement ecclésiastique ${ }^{8}$, sur les congrégations sacerdotales tournées vers l'enseignement ${ }^{9}$ et sur les congrégations féminines de vie active ${ }^{10}$. Parallèlement, les historiens acquéraient une meilleure connaissance de la vie religieuse des laïques. Les exemples les plus probants des nouvelles préoccupations et des résultats obtenus trouvent leurs mesures dans les grandes monographies d'histoire diocésaine ${ }^{11}$.

Puis vint l'histoire des mentalités qui, s'affranchissant de «la problématique institutionnelle», privilégia «une approche davantage anthropologique des faits religieux. Ce changement s'est manifesté à travers le débat sur la religion populaire». L'histoire ainsi renouvelée avait désormais d'autres sujets d'études et, au cœur de celles-ci, se trouvaient les croyances. Les travaux d'Alphonse Dupront autour des sanctuaires s'offrent en exemples: ses enquêtes sur les reliques, les miracles, les ex-voto, la masse des pèlerins sont bien connues ${ }^{12}$. «Toutefois ce sont sans doute les recherches sur la mort et l'au-delà qui ont le plus marqué l'apport de l'histoire des mentalités à l'histoire religieuse $^{13} . »$

Après avoir identifié, trop sommairement il est vrai, les deux approches qui, en quelque sorte, ont renouvelé l'historiographie française, tentons maintenant de voir comment elles furent favorisées par les praticiens de l'histoire religieuse québécoise.

\section{B) SOCIOLOGIE RELIGIEUSE ET RECRUTEMENT}

En 1951, la sociologie religieuse québécoise retardait et JeanCharles Falardeau en attribuait la cause à «l'homogénéité culturelle», à l'enseignement récent de la sociologie et à l'absence d'études sur la pratique religieuse. Fernand Dumont faisait un constat identique quelques années plus tard et il livrait ses vues. Il faisait de la sociologie

8. Pour l'Ancien Régime, voir l'article synthèse de Timothy Tackett, «L'histoire sociale du clergé diocésain dans la France du XVII" siècle», Revue d'histoire moderne et contemporaine (RHMC), 27 (1974): 198-234. Pour la période contemporaine, voir Paul Huot-Pleuroux, Le recrutement sacerdotal dans le diocèse de Besançon de 1801 à 1960 (Paris, Université de Paris, 1966) $517 \mathrm{p}$.

9. Willhem Frijhoff et Dominique Julia, «Les oratoriens de France sous l'Ancien Régime. Premiers résultats d'une enquête», Revue d' histoire de l'Église de France (RHÉF) (1979): 225-265.

10. Claude Langlois, Le catholicisme au féminin. Les congrégations françaises à supérieure générale au XIX siècle (Paris, Cerf, 1984), $776 \mathrm{p}$.

11. Michel Lagrée, «La monographie diocésaine et les acquis de l'historiographie religieuse française», Études d'histoire religieuse (ÉHR), 61 (1995): 9-41.

12. Alphonse Dupront, Du sacré (Paris, Gallimard, 1987), 541 p.

13. Philippe Ariès, L' homme devant la mort (Paris, Seuil, 1977), 642 p.; Michel Vovelle, La mort et l'Occident de 1300 à nos jours (Paris, Gallimard, 1983), 793 p. 
religieuse un instrument de la pastorale et il lui assignait des tâches qui la faisaient largement déborder l'étude de la pratique vers celle des comportements et des représentations afin qu'elle débouchât sur celle de la culture. Un inventaire de Jean-Charles Falardeau, en 1961, révèle que des pas avaient été franchis par la sociologie religieuse ${ }^{14}$, mais il ne dit rien des travaux de Louis-Edmond Hamelin qui s'était inscrit dans la diachronie.

Or, la sociologie religieuse historique est entrée par la porte que celui-ci lui a ouverte. Formé à l'École française de géographie, Hamelin connaissait bien les travaux novateurs de Gabriel Le Bras et ceux du chanoine Boulard qui avait si efficacement illustré une géographie de la différence. Toutefois, Fernand Boulard trouvait que le terme de sociographie convenait mieux à ses propres travaux. C'est aussi ce que Hamelin pense des siens. Avec Colette Hamelin, il publiait, en 1956, Quelques matériaux de sociologie religieuse à propos duquel le père Benoît Lacroix notait dans sa recension l'influence des travaux fran-

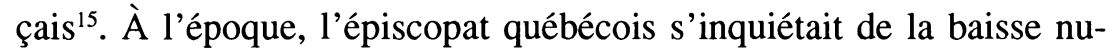
mérique du clergé et c'est cette préoccupation qui inspira le géographe-historien pour diverses contributions. Il traita alors de sujets aujourd'hui classiques: origines sociales et géographiques du recrutement sacerdotal, fécondité sacerdotale, ratio prêtre/fidèles.

Notre connaissance du recrutement ecclésiastique et religieux s'est grandement améliorée depuis les travaux pionniers de LouisEdmond Hamelin sur l'évolution numérique du clergé séculier. Même si sa stratification sociale était «impressionniste», Hamelin a bien observé la fécondité des paroisses plus anciennes. Il reste que les premières recherches systématiques sur les origines sociales du personnel religieux portèrent sur celui de la Nouvelle-France: Micheline D'Allaire traduisit le mode d'appartenance sociale des religieuses en termes de classes dominantes ou de classes dominées ${ }^{16}$, alors que Pierre Hurtubise attribua une origine populaire aux prêtres et aux re-

14. Jean-Charles Falardeau, «Les recherches de sociologie religieuse au Canada», Lumen Vitce (1951): 127-142; Fernand Dumont, «La sociologie religieuse au Canada français», Sociologie religieuse. Sciences sociales. Éditions ouvrières (1955): 150-152; idem, «Sociologie religieuse et pastorale", Ad Usum Sacerdotum, 11,3 (décembre 1955): 67-70; Jean-Charles Falardeau, "Les recherches religieuses au Canada français», Recherches sociographiques (RS) 3,1 (1962): 209-231.

15. Compte rendu de Benoît Lacroix dans la Revue d'histoire de l'Amérique française (RHAF), 12,1 (juin 1958): 130-131. Pour la bibliographie des travaux de Louis-Edmond Hamelin, voir son ouvrage Écho des pays froids (Sainte-Foy, Les Presses de l'Université Laval, 1996), 492 p; voir, en particulier, le chapitre VII, «Études humaines et sociales, des relevés socioreligieux», 299-303.

16. Micheline D’Allaire, «Origine sociale des religieuses de l'Hôpital général de Québec», RHAF, 23,4 (mars 1970): 559-581. 
ligieuses ${ }^{17}$. Pour connaître les caractéristiques sociodémographiques, les chercheurs eurent recours, entre autres sources, aux actes notariés qui ont en commun la dotation d'un patrimoine: la dot des religieuses et le titre clérical des clercs. Largement utilisée en France par les modernistes qui s'intéressaient au bas-clergé, la constitution de la rente patrimoniale ne l'avait pas encore été ici avant les travaux de Pierre Hurtubise, de Jean Roy et de Louise Lebel-Gagnon et Serge Gagnon $^{18}$. L'analyse du recrutement ecclésiastique doit ses autres avancées à l'exploitation des données des recensements. Dès lors, il est possible de poursuivre sur cette lancée et, surtout, nous disposons de points de comparaison fiables et utiles.

Le recrutement des congrégations masculines est largement moins bien connu ${ }^{19}$. Sur ce plan, elles accusent d'ailleurs un grand retard sur les congrégations religieuses féminines. À cet égard, la monographie de Marta Danylewicz ${ }^{20}$ marque d'une pierre blanche ce champ d'investigation, bientôt envahi par les sociologues ${ }^{21}$. Il en ressort les tendances lourdes du recrutement avec, comme raffinement récent, une évaluation de l'apport attendu des groupes sociaux ${ }^{22}$.

Voyons maintenant en termes de résultats. Selon Louise Dechêne, le clergé de Montréal recrutait dans les classes supérieures de la so-

17. Pierre Hurtubise, «L'origine sociale des vocations canadiennes de la NouvelleFrance», Société canadienne d'histoire de l'Église catholique, Rapport (SCHÉC) (1978): 41-56. Hurtubise ne trouva que 16 titres cléricaux. Pour contrer cette obligation, l'Église ordonnait «au service de l'Église» et s'engageait de la sorte à assurer le soutien du futur prêtre.

18. Les articles fondateurs sont de Berthelot Du Chesnay, «Le clergé diocésain français au XVIII e siècle et les registres des insinuations ecclésiastiques», RHMC (1963): $39-43$ et d'André Viala, "Suggestions nouvelles pour une histoire sociale du clergé aux Temps Modernes», dans Études d'histoire du droit canonique dédiées à Gabriel Le Bras (Paris, Sirey, 1965), 2: 1471-1481. L'avantage qu'offre cette source sur l'acte de baptême est certainement la plus grande précision chronologique des informations. Pierre Hurtubise, loc. cit.; Jean Roy, «Le clergé nicolétain, 1885-1904», RHAF, 35,3 (décembre 1981): 383-395; Louise Lebel-Gagnon et Serge Gagnon, «Le milieu d'origine du clergé québécois, 1750-1840: mythes et réalités», RHAF, 37,3 (décembre 1983): 373-393; Gérard Bouchard et Régis Thibault, «Origines géographiques et sociales du personnel religieux dans la région du Saguenay (1882-1947)», Histoire sociale, 55 (mai 1995): 137-157; Gérard Bouchard, Raymond Roy et Pierre Jacques, «La composition des communautés religieuses au Saguenay (1882-1947)», SCHÉC, 55 (1988): 87-119; Christine Hudon, «Carrières et vie matérielle du clergé du Richelieu-Yamaska (1790-1840)», RHAF, 45,4 (printemps 1991): 573-594.

19. Seuls se démarquent Paul-André Turcotte, L'éclatement d'un monde, les clercs de Saint-Viateur et la Révolution tranquille (Montréal, Bellarmin, 1981), 366 p.; et Nive Voisine, Les Frères des Écoles chrétiennes au Canada, 2 tomes (Sainte-Foy, Anne Sigier, 1987, 1991), 443 et $471 \mathrm{p}$.

20. Marta Danylewycz, Profession: religieuse (Montréal, Boréal, 1988), 246 p.

21. Nicole Laurin, Danièle Juteau et Lorraine Duchesne, $\grave{A}$ la recherche d'un monde oublié (Montréal, Le jour éditeur, 1991), $431 \mathrm{p}$.

22. Gérard Bouchard et Régis Thibault, loc. cit. 
ciété $^{23}$. Or, voilà que cette source s'est tarie et que l'Église fut amenée, au lendemain de la défaite de 1760 , à se tourner vers les classes moyennes composées de l'artisanat et des métiers spécialisés les plus lucratifs, ainsi que du côté de la paysannerie aisée. À l'instar de ce qui s'est produit en France après la Révolution, le clergé québécois, d'abord d'origine urbaine, serait ensuite devenu principalement d'extraction rurale, faisant une large part à la paysannerie. Parlerat-on alors de surreprésentation paysanne? Entre 1820 et 1875, les cultivateurs du diocèse de Saint-Hyacinthe contribuaient au recrutement pour une part égale à leur poids démographique ${ }^{24}$. À la fin du siècle, le clergé nicolétain était certainement d'origine rurale sans qu'il y ait là non plus surreprésentation paysanne, alors que celui du Saguenay provient, au $\mathrm{XX}^{\mathrm{e}}$ siècle, d'une manière légèrement privilégiée, de la petite et moyenne bourgeoisie urbaine, sans que l'on puisse cependant dessiner «un modèle préférentiel de recrutement».

Qu'en est-il des religieuses? Existe-t-il un modèle? Marta Danylewicz a conclu à une surreprésentation paysanne chez les sœurs de la Congrégation de Notre-Dame et, plus forte encore, chez les sœurs de la Miséricorde. Même conclusion chez Nicole Laurin, Danielle Juteau et Lorraine Duchesne qui se sont appuyées sur un échantillon couvrant l'ensemble des communautés du Québec pour la période 1841-1971. Enfin, Gérard Bouchard et Régis Thibeault notent que, sous le rapport spatial, le Saguenay paraît se démarquer du reste du Québec par l'absence d'un tel modèle, sauf pour les ursulines de Roberval et les sœurs antoniennes de Marie chez qui les cultivateurs sont représentés en surnombre.

Par ailleurs, pourquoi entre-t-on dans le clergé ou dans une congrégation religieuse? Nous touchons ici à la question fondamentale qui est celle du sens et des motivations. Encore ici, il existe une filiation étroite entre les travaux français et québécois dans l'étude de cet objet particulièrement affectionné par l'École française de la sociologie des religions qui a, notons-le, formé plusieurs des sociologues et historiens québécois des congrégations: Denault, Lévesque, Dussault et Turcotte en sont des représentants. Leurs interprétations sur l'implantation, le développement et le recrutement des congrégations, celles de femmes en particulier, se sont révélées de bons points de départ, même si elles ont été soumises à réinterprétation. Donnons-en les grandes lignes.

23. Louise Dechêne, Habitants et marchands de Montréal au XVII siècle (Paris, Plon, 1974), 477 ss.

24. Christine Hudon, Prêtres et fidèles dans le diocèse de Saint-Hyacinthe, 1820-1875 (Sillery, Le Septentrion, 1996), $469 \mathrm{p}$. 
Pour certains, l'entrée massive dans les communautés aurait été la solution au problème du surnombre des femmes ${ }^{25}$. Pour d'autres, l'expansion des communautés religieuses s'explique plutôt par les aspirations intellectuelles et sociales des femmes. La thèse féministe soutient ici que «l'organisation des couvents par les femmes est la manifestation d'un féminisme naissant» ou d'une «substitution» du féminisme. Dans la plupart des cas, l'explication des motivations procède des facteurs sociaux. L'historienne Marta Danylewycz croit que les hagiographes n'ont abordé ou retenu que les facteurs spirituels, car tout est «attribué au simple désir de se conformer à la volonté divine». Elle pense que l'on ne peut isoler ainsi les deux types de facteurs. De son côté, Nicole Gagnon ${ }^{26}$ fait remarquer que les jeunes filles du début du siècle vivaient dans un contexte de foi qui ne les portait pas à faire un tel choix. Le débat n'est donc pas clos, comme le suggère le titre évocateur: Un métier et une vocation. Le travail des religieuses au Québec de 1901 à $1971^{27}$.

L'histoire des congrégations masculines ne s'est pas construite sur des préoccupations semblables. Elle s'est plutôt intéressée aux raisons de leur implantation au Canada ${ }^{28}$ et, s'interrogeant sur leur pertinence sociale au sein de la société québécoise en voie de sécularisation depuis près de quarante ans, elle s'est préoccupée de leur avenir ${ }^{29}$. L'utopie d'un rassembleur comme le curé Labelle a pu être partagée par des congrégations religieuses françaises en butte aux tracasseries de l'anticléricalisme républicain. En effet, l'explication qui a été donnée récemment à l'immigration congréganiste dans le troisième tiers du XIX ${ }^{e}$ siècle s'accorde bien avec cette utopie de reconquête et d'indépendance politico-culturelle et religieuse, par la réappropriation d'un pays et l'occupation effective d'un territoire ${ }^{30}$.

25. Bernard Denault, «Sociographie générale des communautés religieuses au Québec (1837-1970)», dans Bernard Denault et Benoît Lévesque, Éléments pour une sociologie des communautés religieuses au Québec (Montréal, Les Presses de l'Université de Montréal, 1975), 220 p.; Micheline Dumont, Les religieuses sont-elles féministes? (Montréal, Bellarmin, 1995); un exposé des thèses ainsi qu'une bibliographie pour faire le point.

26. Jean Hamelin et Nicole Gagnon, Histoire du catholicisme québécois. Le XXe siècle, 1: 1898-1940 (Montréal, Boréal, 1984), 504 p.

27. Danièle Juteau et Nicole Laurin, Un métier et une vocation. Le travail des religieuses au Québec de 1901 à 1971 (Montréal, Les Presses de l'Université de Montréal, 1997), 194 p.

28. Guy Laperrière, Les congrégations religieuses. De la France au Québec, 1880-1914, I: Premières bourrasques, 1880-1900 (Sainte-Foy, Les Presses de l'Université Laval, 1996), $228 \mathrm{p}$.

29. Paul-André Turcotte, op. cit.; idem, «L'identité du religieux éducateur dans la mouvance des réformes scolaires au Québec (1920-1990)», ÉHR (1994): 63-84.

30. Gabriel Dussault, Le curé Labelle. Messianisme, utopie et colonisation au Québec, 1850-1900 (Montréal, Hurtubise HMH, 1983), 392 p. 
L'échec de Labelle n'était cependant pas celui de l'Église du Québec dont les fidèles, à l'époque du curé-colonisateur, paraissent afficher des records de pratique pascale. Y aurait-il eu de ce côté une reconquête de la part de l'Église?

\section{C) DÉCHRISTIANISATION APPRÉHENDÉE ET CHRISTIANISATION ENCADRÉE}

La question n'a pas été posée dans ces termes de reconquête, car la problématique de la déchristianisation qui s'est développée en France dans la décennie 1960 n'a pas fait l'objet d'une recherche appliquée au Québec. Et pourtant on a pu conclure que l'Église des débuts du XIX ${ }^{\mathrm{e}}$ siècle luttait pour arrêter la «déchristianisation des campagnes $^{31} »$. Cette conclusion de Jean-Pierre Wallot s'appuyait sur divers témoignages de l'épiscopat et des curés de la seconde moitié du XVIII ${ }^{\mathrm{e}}$ siècle. Il est vrai que le clergé a souvent jeté un regard peu amène sur le comportement des fidèles. Une fois colligés, leurs jugements sévères ont servi à donner une image plutôt sombre du christianisme québécois d'alors. Il me semble que Lucien Lemieux ${ }^{32}$ apporte davantage de nuances, même s'il fonde son analyse sur le prescrit et le vécu. De plus, la triade de Serge Gagnon ${ }^{33}$ sur la mort, sur la sexualité et la confession, et sur le mariage établit une géographie différenciée des mœurs et de la pratique qui n'autorise plus une telle conclusion.

L'histoire comparée révèle avec force l'existence d'un véritable renouveau religieux en Occident à partir des années 1830; ici et là des événements chocs ont favorisé et accéléré l'entreprise d'acculturation par l'Église ${ }^{34}$. Dans cette perspective, les Rébellions de 1837-1838 auraient été l'événement choc qui aurait préparé le terrain aux conversions. Au terme de «renouveau» qui suggère un long travail d'éduca-

31. Jean-Pierre Wallot, «La religion catholique et les Canadiens au début du XIX $\mathrm{X}^{\mathrm{e}}$ siècle», dans Un Québec qui bougeait (Québec, Éditions du Boréal Express, 1973), 210.

32. Lucien Lemieux, «Les années difficiles (1760-1839)», dans Nive Voisine, dir., Histoire du catholicisme québécois (Montréal, Boréal, 1989), 438 p.

33. Serge Gagnon, Mourir, hier et aujourd' hui (Sainte-Foy, Les Presses de l'Université Laval, 1987), 192 p; Plaisir d'amour et crainte de Dieu (Sainte-Foy, Les Presses de l'Université Laval, 1990), 202 p.; Mariage et famille au temps de Papineau (Sainte-Foy, Les Presses de l'Université Laval, 1993), $300 \mathrm{p}$.

34. Claude Langlois, «Permanence, renouveau et affrontement (1830-1880)», dans François Lebrun, sld., Histoire des catholiques en France (Toulouse, Privat, 1980), 335-346; voir les articles de Louis Rousseau et Emmet Larkin, «Le bas clergé catholique au dix-neuvième siècle. Approche comparative d'une population pastorale en voie de changement», dans Louis Rousseau, dir., Les Cahiers de recherche en sciences de la religion, 12 (1995), 355 p; compte rendu de Jean Roy, RHAF, 50,2 (automne 1996): 304-306; Michel Lagrée, «Du bon usage des "chocs" en histoire religieuse», Journées d'études sur la Bretagne et les pays celtiques (1994-1995), 133147. 
tion, devrait-on préférer celui de «réveil religieux» qui connote un changement brusque ${ }^{35}$ ? Nous savons que le sens accordé au «réveil» a été fortement marqué par l'utilisation qu'en ont fait les médiateurs culturels des débuts des années 1840 et les historiens qui, cent ans plus tard, ont désiré célébrer ce qui leur apparaissait comme un événement clé pour comprendre et expliquer la mutation religieuse.

D'autres études se sont ajoutées à celle de René Hardy, qui, en 1968, s'était intéressé à ce problème en l'inscrivant dans la sociologie le brasienne de l'histoire quantitative de la pratique ${ }^{36}$. D'après ces travaux, vers 1875 , plus de $90 \%$ des fidèles se conformaient aux exigences de la pratique pascale; à la fin du siècle, la stricte unanimité d'une paroisse n'était pas chose rare.

On s'entendra bien sur le comment! Mais le pourquoi? Si la conversion explique le changement brusque, peut-elle, à elle seule, expliquer la routinisation? ou bien faut-il recourir à d'autres facteurs, par exemple, l'augmentation des vocations à partir de $1830^{37}$ et l'essor des confréries de dévotion à partir des années $1820^{38}$ ? Tandis que Louis Rousseau relève la césure de 1840 en matière de communion pascale, Brigitte Caulier note le réamorçage de la vitalité confraternelle, avec une accélération dans les années 1840, tandis que la décennie 1850 marque un léger essoufflement du rythme de croissance. Selon Caulier, le retour des laïques vers ces confréries a précédé le réveil religieux. Une autre explication est la démarche plus compréhensive des confesseurs. Mais voilà qui ouvre sur la culture ecclésiastique.

\section{D) LA ROMANISATION DU CLERGÉ ET L'ULTRAMONTANISME RELIGIEUX}

Que sait-on de plus maintenant que n'en disait Pierre Savard, en 1967, sur la vie interne du clergé, sur sa formation et sa spiritualité

35. René Hardy, «À propos du réveil religieux dans le Québec du XIX ${ }^{\mathrm{e}}$ siècle: le recours aux tribunaux dans les rapports entre le clergé et les fidèles (district de Trois-Rivières)", RHAF, 48,2 (automne 1994): 187-212; Louis Rousseau, «À propos du "réveil religieux" dans le Québec du XIX ${ }^{c}$ siècle: où se loge le vrai débat?», $R H A F, 49,2$ (automne 1995): 223-245. Lionel Groulx, «La situation religieuse au Canada français vers 1840», SCHÉC (1941-1942): 51-75.

36. René Hardy, «Note sur certaines manifestations du réveil religieux de 1840 dans la paroisse Notre-Dame de Québec», SCHÉC, 35 (1968): 81-98. Perspective qui ne fut pas relevée par Nive Voisine; néanmoins, il nota la place enfin accordée à l'histoire de la vie religieuse des laïcs: «La production des vingt dernières années en histoire de l'Église du Québec», $R S, 15,1$ (1974): 97-112.

37. Louis-Edmond Hamelin, «Évolution numérique séculaire du clergé catholique dans le Québec», $R S, 2,2$ (1961): 238; l'indice 100 de 1830 donne 1834 fidèles par prêtre; en 1840, l'indice est de 206 et il y a 1185 fidèles par prêtre.

38. Brigitte Caulier, «Les confréries de dévotion traditionnelles et le réveil religieux à Montréal au XIX ${ }^{\mathrm{e}}$ siècle», SCHÉC, 53 (1986): 23-39. 
ainsi que sur sa carrière? Qu'a-t-on ajouté aux traits, à peine esquissés, qu'il lui a donnés et qui apparaissaient comme autant d'orientations de recherche ${ }^{39}$ ? L'historiographie, qui a beaucoup décrit le prêtre comme le chien de garde d'une idéologie ${ }^{40}$, s'est ensuite ouverte aux dimensions pastorales et spirituelles de ce médiateur culturel. Bref, qu'en est-il du bon prêtre ${ }^{41}$ québécois, défini comme un médiateur entre Dieu et l'homme, et entre le monde ecclésiastique et les fidèles ${ }^{42}$ ? Médecin des âmes alors que d'autres sont médecins des corps ou notaires pour répondre à un besoin social, le prêtre est porteur d'une culture savante et d'une culture religieuse qu'il a à charge de transmettre. Il se distingue de la masse des fidèles à qui il s'adresse, d'abord par sa formation religieuse, et aussi par la fonction qu'il assume dans la paroisse, dans l'enseignement et dans la direction des âmes. Sa vision du monde en fait un être à part.

Jugera-t-on exceptionnelle la vision d'Édouard-Charles Fabre, le futur évêque de Montréal? Il est le fils d'un libraire à la foi modérée qui s'opposa puis se résigna à sa vocation. Toujours soutenu par sa mère, le jeune Fabre aima vivre dans la serre chaude du séminaire où la religion l'abritait des effets redoutés de la vie laïque. À ses yeux, la prêtrise était un état bien plus qu'un métier ou une profession. Le prêtre était un appelé et il obéissait à la volonté de Dieu. Sa tâche essentielle était de confesser, tâche lourde que Fabre appréhendait avec frayeur. Mais dans l'Église, il était à l'abri des tempêtes ${ }^{43}$. Il est permis de croire que ses sentiments furent largement partagés par le clergé de l'archidiocèse.

Pour une carrière remarquable comme celle de Fabre, combien d'autres paraissent banales! À suivre ainsi, pas à pas, les membres du clergé, commencent à se dégager des carrières ecclésiastiques dont on voit mieux les réussites et les échecs, faites de promotions et de démotions. Il leur revient de réussir leur carrière, c'est-à-dire de la faire progresser en améliorant leur sort sur tous les plans, dont celui de la vie matérielle. Une cure est bonne par les revenus qu'elle pro-

39. Pierre Savard, «Vue d'ensemble» (1967) et «La vie du clergé» (1966) dans Aspects du catholicisme canadien-français au XIXe siècle (Montréal, Fides, 1980), 15-46.

40. Philippe Sylvain, «Le réveil religieux, (1840-1870)», dans Philippe Sylvain et Nive Voisine, dir., Histoire du catholicisme québécois, II: Réveil et consolidation, 2: 1840-1898 (Montréal, Boréal, 1991), 13-198.

41. Marcel Launay, Le bon prêtre (Paris, Aubier, 1986), 326 p. Un solide point de départ pour une histoire comparée du prêtre paroissial.

42. Michel Vovelle, «Les intermédiaires culturels», dans Michel Vovelle, Idéologies et mentalités (Paris, François Maspéro, 1982), 163-176.

43. François Alary, «Vocation et vision du monde au XIX ${ }^{\mathrm{e}}$ siècle. Le cas de $\mathrm{M}^{\mathrm{gr}}$ ÉdouardCharles Fabre (1839-1846)», ÉHR, 59 (1993): 43-64. 
cure et aussi grâce à la fidélité des paroissiens ${ }^{44}$. Elle se mérite, ce qui se fait en suivant la voie romaine. C'est effectivement en direction de Rome qu'il faut regarder pour trouver d'autres explications au changement religieux, en faisant appel à deux faits indissociables: d'abord l'introduction du liguorisme et sa conséquence qui est la disparition du délai d'absolution qui exerçait une pression sociale intolérable, et ensuite la romanisation du culte que l'on appelle aussi ultramontanisme religieux.

Il y a plus qu'une coïncidence entre le début du renouveau religieux en France, que l'on fait commencer en 1830, et l'introduction du liguorisme qui visait à chasser le rigorisme et sa rigidité, laquelle tenait éloigné de la communion. Les deux premiers évêques de Montréal, Bourget en particulier, ont favorisé son introduction et sa diffusion. Serge Gagnon affirme que, avant 1840, les concours de confession étaient rares. Puis survint «le blitz de confession» de l'aprèsRébellion. Outre la volonté de réconciliation et de pardon, on pourrait certainement y voir, à la suite de Serge Gagnon, l'expression d'une «écoute plus compatissante du pécheur ${ }^{45}$ ».

Mais on ne saurait dire avec précision quand l'enseignement du liguorisme commença à se faire dans les séminaires et à produire les effets recherchés. Son implantation se fit progressivement, car il était sûrement plus difficile de modifier les attitudes enracinées dans une partie du clergé, que d'éduquer de jeunes clercs. Si bien qu'au début des années 1880 , les prédicateurs des retraites ecclésiastiques savaient susciter la réflexion des prêtres sur la position à adopter au confessionnal. Le liguorisme exigeait la sévérité pour le prêtre mais la mansuétude pour le fidèle: «Sois austère pour ta propre conduite, sois indulgent pour celle d'autrui», disait Alphonse de Liguori ${ }^{46}$. Une des conséquences de la nouvelle spiritualité fut de donner une autre image du prêtre, à qui il était demandé de promouvoir le culte ultramontain, de susciter chez les fidèles une pratique plus démonstrative et de leur proposer de nombreuses dévotions, inscrites dans un calendrier chargé. L'expression de «populisme religieux ${ }^{47}$ » prend ici son sens.

44. Frank W. Remiggi, «La gestion épiscopale du personnel pastoral dans la grande région de Montréal, 1820-1880», et Louis Rousseau et Pierre Feuvrier, «Morphologie des carrières sacerdotales dans le Sud-Ouest québécois», dans Louis Rousseau, dir., loc. cit., 117-150; 191-209.

45. Serge Gagnon, Plaisir d'amour et crainte de Dieu (Sainte-Foy, Les Presses de l'Université Laval, 1990), 101.

46. Rodrigue Théberge, «Une morale pour une pastorale de la miséricorde. L'homo apostolicus», dans Jean Delumeau, dir., Alphonse de Liguori, pasteur et docteur (Paris, Beauchesne, 1987), 131.

47. Michel Lagrée, «Religion populaire et populisme religieux au XIX ${ }^{\mathrm{e}}$ siècle», dans Jean Delumeau, dir., op. cit., 157-178. 
En somme, le monopole clérical de la religion au XIX ${ }^{\mathrm{e}}$ siècle s'est construit sur la nécessaire solution du problème posé par les délais d'absolution et la soumission à l'exigence de la communion pascale, et sur la propriété unique des moyens de salut. Religion de clercs, alors?

\section{E) LA RELIGION POPULAIRE}

Le père Benoît Lacroix marquait son intérêt pour l'étude du vécu religieux en mettant sur pied, en 1967, le Centre d'études des religions populaires et en instituant une série de colloques sur ce thème. L'objet était beau et invitant, mais non défini. Le mot «populaire» ne plaisait pas beaucoup à Fernand Dumont qui disait craindre la création d' «une confusion, même d'une opposition entre la vie religieuse courante et la vie religieuse de la théologie ${ }^{48}$ ». Quelque cinq ans plus tard, au colloque de Sherbrooke (1973), Jean Delumeau faisait remarquer qu'il ne faisait pas usage du mot populaire parce que les sources qu'il utilisait étaient l'écrit: «Car, expliquait-il, quand on croit l'atteindre, on demeure prisonnier de la civilisation de l'écrit qui ne laisse passer la culture des masses qu'en la filtrant et en la colorant de ses préoccupations.» De son côté, Jean Séguy se montrait tout aussi prudent et mettait de nouveau en garde contre le concept non critiqué de religion ou de dévotion populaire, ainsi qu'il l'avait d'ailleurs fait aux premier et troisième colloques sur ce thème.

Bien qu'une étude de Gérard Bouchard ait soulevé de très sérieuses réserves sur ce concept, lors du colloque de $1975^{49}$ qui se déroula à Chicoutimi, c'est en France que le débat se fit. En 1977, on attendait encore de la religion populaire qu'elle fasse les preuves de sa qualité à «produire de l'histoire ${ }^{50}$ ». N'était-elle enfin qu' «une fausse porte ouvrant sur des recherches fécondes?», de se demander Marc Venard $^{51}$. Pierre Boglioni ne pensait sans doute pas autrement en préfaçant l'ouvrage de Marie-Aimée Cliche sur Les pratiques de dévotion

48. Giselle Huot, dir., Dits et gestes de Benoît Lacroix, prophète de l'amour et de l'esprit (Saint-Hippolyte et Montréal, Éditions du Noroît et Fondation Albert-le-Grand, 1995), 221-257, pour l'origine et la vie du Centre d'études des religions populaires. Plus particulièrement, le texte de Guy Laperrière, «Comment s'organisaient les colloques des religions populaires», 232-237, est éclairant; l'opinion de Fernand Dumont est tirée d'une lettre du 21 février 1968, 229.

49. Andrée Désilets et Guy Laperrière, dir., Recherche et religions populaires (Montréal, Bellarmin, 1976), 15 et 35, colloque international, 1973; Gérard Bouchard, «Sur l'Église catholique et l'industrialisation au Québec: la religion des Eudistes et les ouvriers du bassin de Chicoutimi, 1903-1930», Protée, 4 (1976): 31-42.

50. Claude Langlois, «Sociologie religieuse historique et religion populaire», dans $\mathrm{La}$ religion populaire, colloque international du CNRS (Paris, 1979), 334.

51. Marc Venard, compte rendu du Colloque international de Sherbrooke, dans RHÉF, 64,172 (janvier-juin 1978): 111. 
en Nouvelle-France ${ }^{52}$. Selon lui, deux composantes décisives devaient entrer dans sa définition: une référence claire aux couches populaires dans lesquelles cette religion s'incarnait, et une attention explicite aux différences et tensions entre cette religion et la religion officielle. En dehors de ces deux dimensions, écrivait-il, la perspective de la «religion populaire perd son originalité et sa fécondité propres». Et il trouvait préférable d'abandonner l'utilisation du concept dans les cas des «sociétés [...] acculturées par la religion cléricale», comme on avait pu l'observer dans la paroisse du bassin de Chicoutimi où, «toujours, le clergé paroissial proposait les formules et les cadres ${ }^{53} »$.

Mettant à profit les cahiers de prônes et les bulletins paroissiaux, une semblable lecture fut faite par plusieurs à propos de diverses paroisses du diocèse de Trois-Rivières et de la paroisse de SaintPierre-Apôtre de Montréal ${ }^{54}$. «Religion populaire, religion de clercs?» a-t-on demandé. La réponse était évidemment religion de clercs: ceux à qui justement appartenait l'initiative ${ }^{55}$. Ici, l'institution ecclésiale recouvre entièrement la religion du quotidien ${ }^{56}$ : religion qui se détache sur un fond d'habitudes. Mais qu'en est-il de «l'extraordinaire» qui est fait, ainsi que le dit Isambert, d'une rupture du rythme par des gestes exceptionnels tels le pèlerinage, les cultes thérapiques, les dévotions dont la visée est le miracle, ou au moins les manifestations d'efficacité directe du sacré? Or, ajoute-t-il, ces pratiques tendaient à être encadrées, modérées, limitées et, par là même, partiellement combattues par l'Église. Cette lecture me paraît s'appliquer à la situation québécoise. Voyons cela à travers deux exemples séparés par autant de siècles: le premier est fourni par les travaux de Marie-Aimée Cliche, le second est de Jean Roy.

La vigilance du clergé de la Nouvelle-France, dit Cliche, s'est particulièrement exercée à l'endroit des manifestations les plus spontanées des sentiments religieux. Pour obtenir la satisfaction de faveurs temporelles ou spirituelles, on recourait à l'intermédiaire des saints et

52. Marie-Aimée Cliche, Les pratiques de dévotion en Nouvelle-France (Sainte-Foy, Les Presses de l'Université Laval, 1988), 353 p. Préface de Pierre Boglioni, vii-xix.

53. Gérard Bouchard, loc. cit., 41.

54. Serge Gagnon et René Hardy, L'Église et le village au Québec, 1850-1930 (Léméac, 1979), 171 p.; Lucia Feretti, Entre voisins (Montréal, Boréal, 1992), 264 p.

55. Benoît Lacroix et Jean Simard, dir., Religion populaire, religion de clercs? (Québec, Institut québécois de recherche sur la culture, 1984), 439 p. Le titre, inspiré de la communication de Guy Laperrière, paraît plutôt révélateur et peut se présenter comme une conclusion au demier des colloques sur la religion populaire, en 1982.

56. François-André Isambert, Le sens du sacré, fête et religion populaire (Paris, Les éditions de Minuit, 1982), chapitre 1, «La religion populaire, mythe ou réalité?», 22-25. Dans cette première partie qu'il a intitulée «La religion populaire fraiche et joyeuse», qu'il applique à Dupront, l'auteur critique la conclusion que celui-ci a donnée au colloque de 1977. 
des reliques. $\mathrm{Si}$, par miracle, l'effet désiré se produisait et, surtout, si le récit qui en était fait apparaissait «digne de foi» au clergé, celui-ci pouvait penser à l'utiliser pour augmenter la piété. Cela exigeait cependant qu'il le reconstruise à la mode tridentine: Dieu y apparaissait habituellement comme l'auteur du prodige, le saint comme simple intermédiaire, les objets de piété comme des moyens de manifester sa dévotion ${ }^{57}$.

Point de miracle à Saint-Célestin où l'évêque a favorisé l'établissement d'un pèlerinage diocésain à partir d'une chapelle privée et, à vrai dire, assez peu fréquentée depuis le décès de son propriétaire et ancien curé de la paroisse. En ce premier quart du $\mathrm{XX}^{\mathrm{e}}$ siècle, La Tour des Martyrs ${ }^{58}$ donne l'exemple d'une création plutôt artificielle mais aussi d'un lieu de pèlerinage bien organisé, dédié à sainte Anne et aux saints et où l'on offre des milliers de reliques à la dévotion des visiteurs. Mise à part cette particularité qui n'est pas que curiosité, cette invention du clergé prend place parmi de nombreux autres lieux de pèlerinage. Cette initiative du clergé souleva chez ses membres des sentiments variables qui allèrent de la sympathie à l'indifférence ou encore au scepticisme quand, en 1953, on les interrogea sur la continuité à donner à cette œuvre. Cette religion avait vécu.

$$
* * *
$$

À un relevé exhaustif, quasi impossible, fut préféré un bilan qui a pris la forme d'une démonstration de la dynamique de la recherche et de la progression des résultats dans quelques domaines seulement. J'ai donc choisi, par exemple, de ne rien dire de la production historiographique de l'Église - institution qui, dans la première moitié du XIX ${ }^{\mathrm{e}}$ siècle, innove pour s'adapter à la réalité de la soumission politique et pour se doter des moyens de sa mission. Ce n'est pas un des moindres apports de Lucien Lemieux ${ }^{59}$. Fut également passé sous silence l'Action sociale de l'Église au XX' $\mathrm{X}^{\mathrm{e}}$ siècle. J'ai plutôt opté pour quelques-uns des faits qui, me semble-t-il, ont structuré l'histoire du catholicisme québécois: le personnel religieux et, ensuite, la centralisation romaine, cet autre impérialisme disait Fernand Dumont, qui a

57. Marie-Aimée Cliche, op. cit., chapitre II, «Les pratiques de piété», 13-74; sur les miracles, on consultera son article «Dévotion populaire et encadrement clérical en NouvelleFrance: la croyance au miracle dans la région de Québec», SCHÉC (1985), 17-34.

58. Jean Roy, «L'invention du pèlerinage de La tour des Martyrs de Saint-Célestin (18981930)», RHAF, 43,4, (printemps 1990): 487-507.

59. Voir la note 32. 
donné ses caractéristiques ainsi que son contenu à la culture religieuse des clercs et des laïques.

L'historiographie de ces objets, entourés de débats, a une inscription sociale. Ainsi, France, pays de mission des abbés Godin et Daniel $^{60}$ résonna comme un coup de tonnerre et souleva des interrogations à propos de la pastorale que la sociologie religieuse rétrospective prit bientôt à son compte. Au Québec, au milieu de la décennie 1950, sociologie et pastorale était également un thème cher à des universitaires, dans un contexte où des membres du clergé établissaient des liens entre l'industrialisation et l'affaiblissement de la pratique religieuse. Le relais fut pris, en France, par l'étude de la déchristianisation et, de ce côté-ci de l'Atlantique quoique plus tardivement, par l'étude de la religion populaire, grâce encore cette fois à un autre membre du clergé, Benoît Lacroix. Traditionnelle et rurale, la religion populaire en question faisait déjà l'objet d'enquêtes de la part des ethnographes prédécesseurs des historiens sur ce terrain qui n'avait pas même «La Beauté du mort ${ }^{61} »$. L'inscription sociale des études de l'implantation des congrégations religieuses de femmes et de leur rôle n'est pas moins nette, tant ces recherches sont redevables aux études féministes et aux sociologues qui ont défriché ce terrain avant les historiens.

L'approche comparatiste s'est ensuite imposée à la recherche universitaire du fait même du catholicisme et de son évolution, en France notamment. Elle aura permis d'enrichir plusieurs problématiques dont celles du recrutement sacerdotal et du renouveau religieux. Nourri à celles de la sexualité, de la mort et de la confession, Serge Gagnon, par exemple, aura beaucoup ajouté aux connaissances. Mais qui désire faire le point sur les mérites de l'approche comparatiste, de son utilité, de son usage qui n'est pas que récent, trouvera une matière abondante dans Prêtres et fidèles dans le diocèse de Saint-Hyacinthe, 1820-1875, de Christine Hudon. Il sera en mesure d'apprécier une monographie dont il est permis de dire qu'elle se rapproche beaucoup, quoique sans y tendre, de l'approche diocésaine française.

60. Henri Godin et Yvon Daniel, La France, pays de mission (Paris, Cerf, 1943), 161 p.

61. Michel de Certeau, Dominique Julia et Jacques Revel, «La beauté du mort», dans Michel de Certeau, La culture au pluriel (Paris, Union générale d'édition, 1974), 55-94. 